\title{
DECIPHERING THE ACTION MECHANISM OF INDONESIA HERBAL DECOCTION IN THE TREATMENT OF TYPE II DIABETES USING A NETWORK PHARMACOLOGY APPROACH
}

\author{
PETER JUMA OCHIENG ${ }^{\mathrm{a}}$, WISNU ANANTA KUSUMAa,c, MOHAMAD RAFI ${ }^{\mathrm{b}, \mathrm{c},}$, TONY SUMARYADA ${ }^{\mathrm{d}}$
}

aDepartment of Computer Science, Bogor Agricultural University, ${ }^{b}$ Department of Chemistry, Bogor Agricultural University, ${ }^{c}$ Tropical Biopharmaca Research Center, Bogor Agricultural University, (IPB), Jl. Taman Kencana No. 3, Bogor 16128, Indonesia, dComputational Biophysics and Molecular Modeling Research Group (CBMoRG), Department of Physics, Bogor Agricultural University, Kampus IPB

Dramaga, Bogor 16680 Indonesia

Email: peter_ochieng@apps.ipb.ac.id

Received: 30 Nov 2016 Revised and Accepted: 30 Jan 2017

\section{ABSTRACT}

Objective: The aim of this research was to investigate action mechanism of Indonesia herbal decoctions in the treatment of Type 2 Diabetes (T2D) using network pharmacology approaches.

Methods: Drug target profile analysis via Markov clustering was performed to identify the potent antidiabetic ingredients in the four herbs. Network target base identification of multicomponent synergy was applied to predict the ingredients synergetic effect. The multi-level and integrated target networks were contracted to identify the herbs major ingredients and their presumed targets. Further enrichment analysis and molecular docking were performed to validate network targets.

Results: 278 ingredients from the four herbs were linked to antidiabetic drugs with an overall clustering success rate of $98.58 \%$ and 5 ingredient pairs had significant synergetic effects. Enrichment analysis demonstrates herbs candidate presumed targets were frequently involved in the significant biological process and pathways associated with progression of Type 2 diabetes (T2D) diseases. Finally, molecular docking validation revealed there was high binding site similarity between momordicoside F2 (78\%), beta-sitosterol (67\%) and cis-N-Feruloyltyramine (67\%) with miglitol drug. In addition, the four ligands presented the higher binding affinity to Maltase-glucoamylase (MGA) receptor an enzyme responsible for the digestion of dietary starch to glucose.

Conclusion: This study revealed the pharmacological mechanism of action of Indonesia herbal decoctions in the treatment of Type 2 diabetes. The herbs major presumed target played a significant biological role in the progression of Type 2 diabetes (T2D) while major herbal ingredients indicates the potential of curing Type 2 diabetes by inhibiting Maltase-glucoamylase (MGA) activity.

Keywords: Type 2 diabetes, Indonesia herbal decoction, Network pharmacology

(C) 2016 The Authors. Published by Innovare Academic Sciences Pvt Ltd. This is an open access article under the CC BY license (http://creativecommons.org/licenses/by/4.0/) DOI: http://dx.doi.org/10.22159/ijpps.2017v9i3.16413

\section{INTRODUCTION}

Type 2 diabetes (T2D) or noninsulin-dependent diabetes mellitus (NIDDM) is a chronic metabolic disease characterized by elevated blood glucose level due to insufficient insulin secretion, insulin resistance or insulin impairment $[1,2]$. International Federation of Diabetic (IFD) estimated 90\% of 285 million people suffering from the diabetic disease are diagnosed with Type 2 diabetes [3]. Epidemiological studies have reported genetic and environmental factors might be the possible contributing factor to the loss of beta cell function in Type 2 diabetes patients [4]. This leads to impairment of insulin action and secretion causing hyperglycemia a condition characterized by glucotoxicity that marks the onset of Type 2 diabetes complications [5-7]. Therefore, to regulate glycemic homeostasis in Type 2 diabetes patient's synthetic drugs such as metformin, alpha-glucosidase inhibitors, sulfonylureas, thiazolidinediones (TZDs), and insulin injections are often used [8-10]. Some of those drugs have indicated therapeutic activity to regulate blood glucose level. However, others have shown low efficacy with various side effects associated with flatulence and diarrhea in Type 2 diabetes patients [11-13].

Thus to overcome such side effects associated with synthetic drugs, herbal medicines are often used as the alternative drug for treatment of Type 2 diabetes [14]. Ingredients in herbal plants such Momordica charantia have indicated therapeutic activity by regulating the blood sugar level in diabetic mice and improving insulin resistance and hyperlipidemia in rats [15]. Blumeatin from Blumea balsamifera to have indicated antihyperglycemic effects on diabetic rats while berberine from Tinospora cordifolia has been reported to be effective in reducing blood glucose by enhancing insulin receptor expression [16-18]. Ingredients such gingerol and shogaol from Zingiber officinale have indicated to some activities of increasing insulin receptor signaling [19]. Despite those herbs indicating therapeutic activities on Type 2 diabetic, their mechanism of action remains unknown due to their numerous complex mixture that often bound transiently to multiple targets [20-22].

In drug discovery, network pharmacology paradigms are often applied to understand the underlying pharmacological mechanism of action of a given drug to certain disease [23-26]. Recently, omic technologies and systems biology have been adopted to predict the combinatory drug effect in order to understand their pharmacological mechanism based on target analysis [27]. Furthermore, in networks pharmacology, both technologies are often integrated to reveal the relationship between drugs and their targets [28]. Basically, there are two types of the network pharmacology techniques; a bottom-up approach which entails the addition of well-known molecular drugs targets and the observed synergistic effects and the top-down approach which is a general reduction of given formula to the minimal elements but still maintain important properties [29].

The aim of this paper, we developed a comprehensive systematic approach to investigate the pharmacological mechanisms of action of Indonesia herbal decoction (Zingiber officinale, Tinospora cordifolia, Blumea balsamifera and Momordica charantia) in the treatment of Type 2 diabetes. The protocol of our study includes; (i) Prediction of antidiabetic ingredients in the four Indonesia herbal decoction using drug target profile analysis via Markov clustering algorithm. (ii) Synergy prediction among the ingredients in the four herbs by network target base identification of multicomponent 
synergy approach. (iii) Identification of candidate major presumed targets and their corresponding herbal ingredients by multi-level and integrated network target technique. (iv) Enrichment analysis for validation of the biological significance of major presumed targets as well as molecular docking validation of binding affinity major corresponding herbal ingredient.

\section{MATERIALS AND METHODS}

\section{Materials}

The herbal ingredient data were obtained from IPB University 'jamu' Knapsack (http://jamu. ipb. ac. id) and other reliable literature on related herbal ingredients. Herein, 286 ingredient compounds (37 Tinospora cordifolia, 71 Zingiber officinale, 35 Momordica charantia, and 143 Blumea balsamifera) were investigated for their antidiabetic properties. The chemical information on herbal ingredients such as structure, canonical names and chemical identity (CID) were downloaded from PubChem compound database [30, 31]. The genes associated with Type 2 diabetes and therapeutic targets Food and Drug Administration (FDA) drugs were downloaded from OMIM Morbid Map [32] and DrugBank databases [33], respectively. PPI data were imported from four existing PPI databases, namely the Human Annotated and Predicted Protein Interaction Database (HAPPI) [34], Online Predicted Human Interaction Database (OPHID) [35], Human Protein Reference Database (HPRD) [36], Molecular Interaction Database (MINT) [37].

\section{Identification of antidiabetic herbal ingredients}

The antidiabetic ingredients from the four herbs were identified by network drug target profiles analysis via Markov clustering (MCL) algorithm. Herein, Food and Drug Administration (FDA) approved antidiabetics drugs (rosiglitazone, pioglitazone, repaglinide, tolrestat, miglitol, rimonabant, praminitide, phenformin and starsis) were used to construct ingredient-drug target network. The network was then partitioned using Markov clustering (MCL) algorithm to generate ingredient-drug target profiles. The optimal inflation and cutoff parameters for MCL algorithm were set to generate maximum cluster granularity. The reliability of the MCL algorithm was assessed by determining the misclassification rate: the number of misclassified antidiabetic ingredients/a total number of identified antidiabetic ingredients in other words when applicable the overall success rate: the fraction of times the MCL algorithm recovered the correct number of clusters $\times$ (1-the misclassification rate).

\section{Determination of synergetic effect among the herbal ingredients}

The ingredient synergetic effects were computed by network target based identification of multicomponent synergy (NIMS). In the proposed method, a set of genes or gene products affected by an agent (herbal ingredient) is termed agent genes, and the diseasespecific biological network serves as the background network to compute the synergy scores. Thus, the two elements 'topology score' (TS) and 'agent score' (AS) were used to evaluate ingredient interactions [38]. Topological scores were calculated from topological features of the Type 2 diabetes (T2D) disease network. From the network target perspective, the Achilles 'heel of the biological network underlying a certain disease is more likely to become the attack points of herbal ingredients [39]. Therefore, we assume that the more important the agent gene as a network node is, the stronger synergetic effect the agent will produce the Type 2 diabetes disease. Thus significant synergetic scores of the agent gene as a node in the network were computed based on the node importance values by integrating degree, betweenness, and closeness as the three network centrality indexes to measure the network properties of ingredient targets [40,41]. Note that for synergy prediction we hypothesize that if an ingredient pair produces synergy, their agent genes should be adjacent in the network.

\section{Network construction and analysis}

The herbs, chemical ingredients, presumed targets and known therapeutic targets of Type 2 diabetes were used to construct protein-protein interaction (PPI) network (presumed targets), a multi-level interaction network of herb-chemical ingredients- presumed target, lastly an integrated herb-chemical ingredientpresumed target-known T2D therapeutic target network to understand the relationship between the herbs and their presumed targets. The PPI data were obtained from existing PPI databases mentioned in materials section then Cytoscape visualization software (Version 2.8.1, Boston, MA, USA) was the used to visualize the network [42].

\section{Defining network topological features set}

For the purpose of understanding of the relationship between the herbs, their ingredients and presumed targets as well as their therapeutic targets we defined the network topological features by considering reference node in the network to be node $i$ and measured four main topological features. (i) Degree the number of edges connected to node i. (ii) Node betweenness the number of the shortest paths between two of nodes intersecting node i. (iii) Closeness the inverse of the farness or sum of the node i distances to all other nodes. The closeness centrality is also considered as a measure of how long it will take to sequentially spread information from node i to all the other nodes [43]. Based on that we used the degree, node betweenness, and closeness centralities to measure topological importance in our network, thus the larger degree or node betweenness or closeness centrality, the more important that ingredient, protein or therapeutic target in the network. (iv)The kcore analysis is an iterative removal of least connected nodes from the networks. The maximum order of the core is defined as the highest k-core of the network enhance ' $\mathrm{K}$ value' is used to measure the centrality of node i in the network [44].

\section{Herb-chemical ingredients-presumed target-known T2D target network}

The herb-chemical ingredient-presumed target-known T2D therapeutic target network was constructed by linking the four herbs their chemical ingredients, presumed targets and the known T2D therapeutic targets that interact with the presumed targets. Herein we consider the node was as a hub protein if its degree was more than 2 -fold the median degree of all the nodes in a network. Then, the PPIs among the hub protein targets were used to construct the hub presumed target PPI network. We used four network topological features such as 'Node degree', 'Closeness', 'Node betweenness', and 'K value' previously defined in the feature set definitions to identify the major presumed targets, in case the four topological features sets have values higher than the corresponding median values. We further to generate a k-core network of the original integrated network by iteratively removing the least connected nodes from the network whose degree is less than $\mathrm{k}$. Performing k-core analysis, we obtain a sub-network, which is a globally central region of the original network.

\section{Enrichment analysis of herbs major presumed targets acting on T2D disease}

The enrichment analysis based on PANTHER functional annotation system [45] and Diversity Visualization Integrated Database (DAVID) [46] was performed to validate the biological significance of major presumed targets with the $\mathrm{P}$ value $<0.05$ after Benjamini's correction. A higher score indicates that the gene members are involved in more important (enriched) biological processes.

\section{Molecular docking of herbs major ingredients}

The binding affinities of the major herbal ingredients were validated by molecular docking using Autodock Vina software [47]. The Maltase-glucoamylase receptor (MGA) and major herbal ingredients (ligands) were selected based on their topological importance from the k-core sub-networks. The structures of the receptor (Maltoseglucoamylase with PDB code 2QMJ) and the four ligands (betasitosterol from the Zingiber officinale with CID code 222284, cis-NFeruloyltyramine from Tinospora cordifolia CID code 5280537, linderol from Blumea balsamifera with CID code 65373, and momordicoside F2 from Momordica charantia with CID code 44445567) were downloaded from PubChem website. Both the ligands and receptor were initially prepared using Autodock Tools program [48]. For the empirical comparison, we used miglitol (also known as Glyset, a patented drug that capable of inhibiting the 
alpha-glucosidase activity) as control ligand. All structures for receptor and ligands were saved in a. pdbqt format to meet the docking program requirement. The next step we set the grid coordinates as $\mathrm{x}=-27.643, \mathrm{y}=-12.49$ and $\mathrm{y}=-0.559$ and the box size set as $\mathrm{z}=30, \mathrm{y}=30$ and $\mathrm{x}=36$, as well as the number of exhaustiveness, was set at 20 to compromise between the exhaustive search and global minimum for better docking results. The binding sites regions were visualised by LigPlot+v.1.4 program [49].

\section{RESULTS AND DISCUSSION}

\section{Identification of antidiabetic herbal ingredients}

The antidiabetic ingredients from the four herbs were determined based on the hypothesis that chemical ingredient in the four herbs and Food and Drug Association (FDA) approved antidiabetic drugs were likely to share similar target profiles, biological function, and pharmacological action as well as the mechanism of action. According to target profile analysis (fig. 1), 22 ingredients from zingiber officinal; 25 ingredients from Momordica charantia; 13 ingredients from Tinospora cordifolia and 82 ingredients from Blumea balsamifera were identified from the clusters and were linked to known Food and Drug
Association (FDA) antidiabetic drugs, this reveals that certain ingredients compounds were shared diverse pharmacological action with targeted antidiabetic drugs.

Some of the identified ingredients were linked with diverse antidiabetic activities in previous studies for instance 5,7,3',5'tetrahydroxy flavanone and blumeatin from Blumea balsamifera to have been reported to be antioxidants that improve diabetes; guaifenesin from Tinospora cordifolia enhance the neurite outgrowth which might protect Type 2 diabetes (T2D) patients from neuropathy [50]. For instance, jatrorrhizine, berberine bisulfate, coptisine, epiberberine, oxyberberine, columbamine, and berberine were reported to have hypoglycemic and antidiabetic actions that regulate glucose metabolic effect and reduction of oxidative stress injury $[51,52]$.

The results obtained in target profile analysis revealed our proposed Markov clustering (MCL) to be more effective for identification of antidiabetic compound in the four herbs with an overall success rate of $98.58 \%$. This demonstrated the reliability of our approach to provide a possible explanation for action mechanism and molecular basis of the four Indonesia herbs in the treatment of Type 2 diabetes.
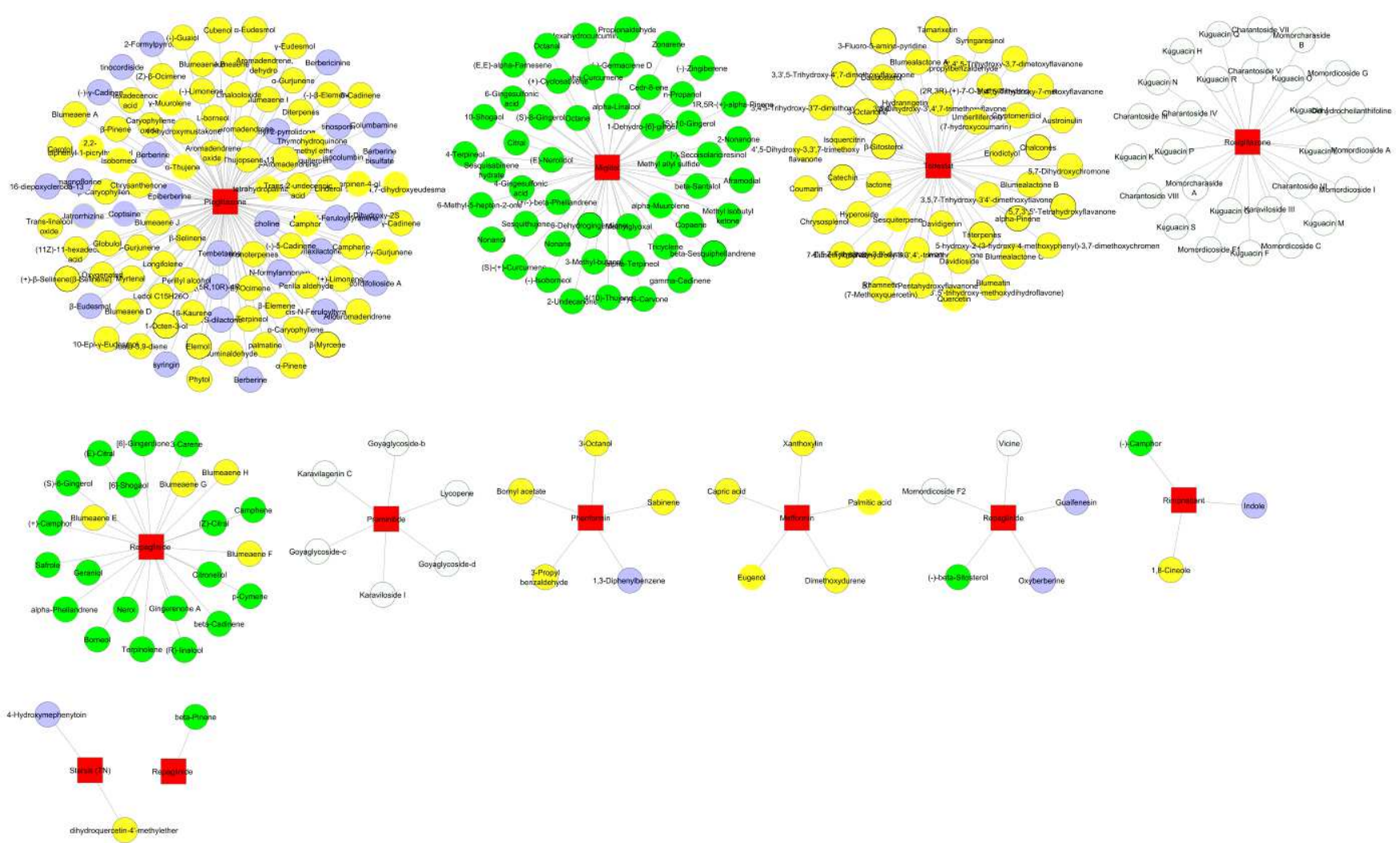

Fig. 1: The herbal ingredient-drug networks based on the target profile cluster analysis. Edges: the link between the herbal ingredients and FDA drugs; red square nodes: known FDA antidiabetic; the circle nodes herbal ingredients; green nodes: Zingiber officinale; blue nodes: Tinospora cordifolia; yellow nodes: Blumea balsamifera; barge node: Momordica charantia

\section{Synergetic effect among herbal ingredients}

For synergy prediction, berberine was used as the core agent (ingredient) due to its antidiabetic activity [53]. In the proposed method, a high score means a great probability of synergy and we measure the synergy of ingredients combination with independent mechanisms based on Bliss independent theory [54], so we roughly set the valid range of our score from 0 to 0.9 . From the network, 16 unique berberine-ingredient synergy scores were obtained and combination of berberine with momordicoside F2 (0.3976) from Momordica charantia, linderol (0.3071) from Blumea balsamifera and beta-sitosterol (0.4332) and (S)-10-Gingerol (0.4129) in Zingiber officinale as well as cis-N-Feruloyltyramine (0.3711) in
Tinospora cordifolia presenting significant antidiabetic synergetic effect. Those synergetic effects might be due to their deferent antidiabetic mechanisms as the cluster analysis revealed those ingredients to have deferent target profiles. We further validate the synergy scores by comparing with Food and Drug Association (FDA) approved antidiabetic drugs we used in target profiles analysis. Related studies have reported the combination of repaglinide and pioglitazone to have an acceptable safety with greater reductions in glycemic parameters than treatment using either agent alone [55]. The proposed method demonstrates that the network target can nicely interpret the synergetic mechanism of ingredients combination in Indonesia herbal decoction by its latent network topology properties. 


\section{Herb-chemical ingredients-presumed target network}

The multi-level network of herb-chemical ingredients-presumed target revealed the relationships between the herbs and their presumed targets. The network (fig. 2) consists of 862 nodes (4 ingredients, 282 chemical ingredients, and 576 presumed targets) and 1227 edges. The mean number of presumed targets per chemical ingredients was 2.042 . Among 282 chemical ingredients, 7 had high-degree distributions, and each hitting significant presumed targets two of them come from Tinospora cordifolia, such as 4-
Hydroxymephenyton and mangnoflorine; two from Zingiber officinale, such as 1,3-cineole and (-)-beta sitosterol and monordicoside F1 from Momordica charantia as well as alphapinene and linderol from Blumea balsamifera. From the four herbs, Blumea balsamifera had the highest degree distribution, hitting 33 presumed targets. Network analysis reveals 10 presumed targets were linked to Tinospora cordifolia, Zingiber officinale, and Momordica charantia herbs and 24 presumed targets were shared with among the four herbs this explains the functional relationship between herbs their ingredients and presumed targets.

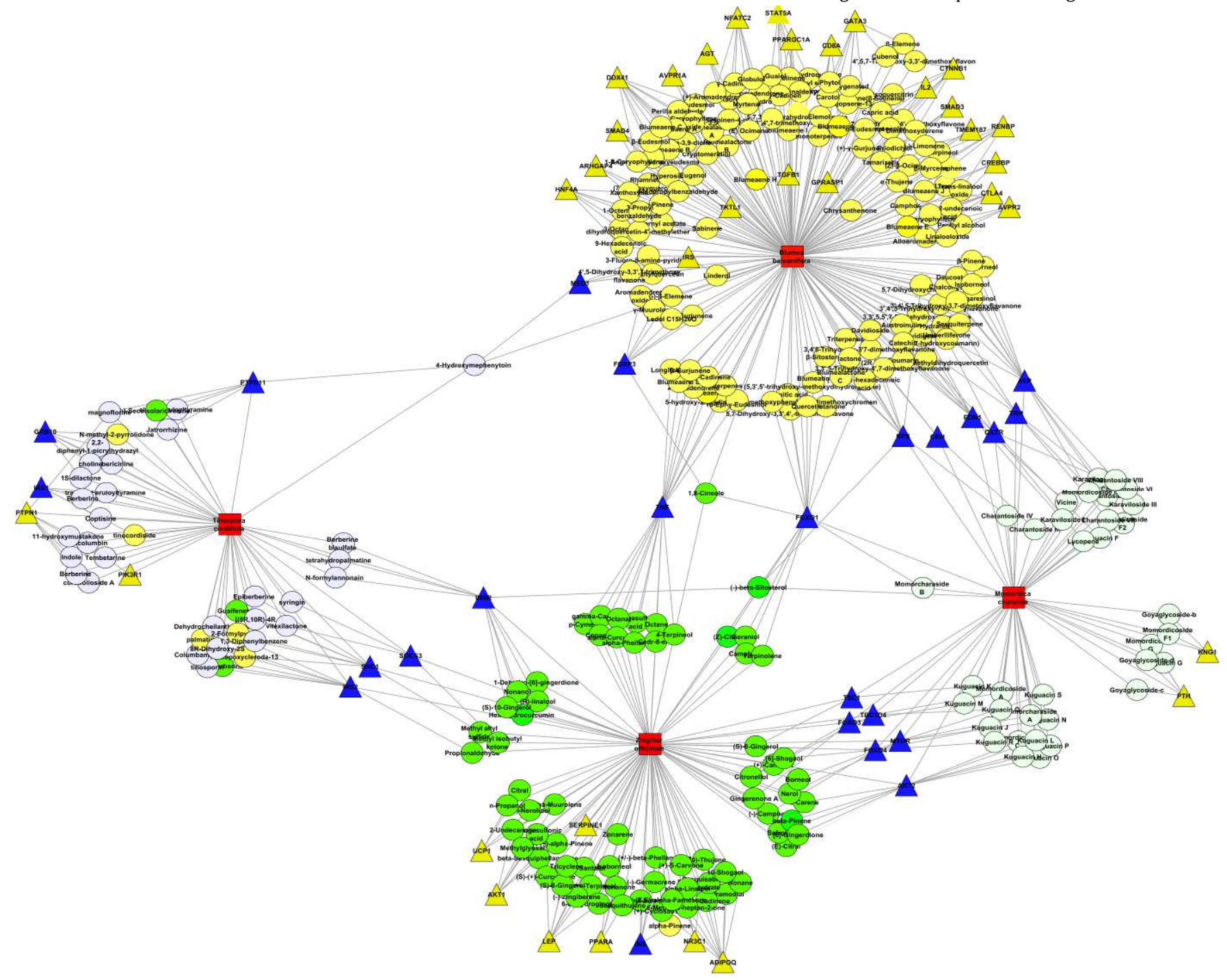

Fig. 2: Interaction network to understand the relationship among herbs, their ingredients and presumed targets visualized by Cytoscape. Edge; interaction between ingredients and their presumed targets; red rectangle node: Herbs, including Tinospora cordifolia, Zingiber officinale, Momordica charantia and Blumea balsamifera; circle nodes: Herbal ingredients; brown triangle nodes: Presumed targets for herbal ingredients; blue triangle: Presumed targets shared by the four herbs

\section{Herb-chemical ingredient-presumed target-known T2D therapeutic target network}

The integrated network (fig. 3A) built by reversed imbalanced herbingredient-presumed target-known Type 2 diabetes (T2D) target network to understand the mechanism of action of the four herbal ingredients in the treatment of Type 2 diabetes (T2D). From the network, we identified 871 nodes (including 4 herbs and 282 ingredients, as well as 576 presumed targets and 9 known therapeutic targets) with 1227 interactions. Based on the four topological features describe in materials and methods section we identified 54 major nodes linked to 31 ingredients contained in the four herbs, 24 presumed targets were shared, and 2 known therapeutic targets had the highest degree distribution furthermore, all the nodes had significant degree, betweenness, closeness and $\mathrm{K}$ value greater than the corresponding median values. To identify the herbs major presumed targets and ingredients acting on Type 2 diabetes we performed the K-core analysis (fig. 3 B) by iteratively removing less connected nodes to obtain the global central region of original network (fig. $3 \mathrm{~A}$ ). From the k-core sub-network, 68 nodes (31 chemical ingredients, 24 presumed targets, and 2 major T2D therapeutic targets) and 132 interactions were identified.

From the sub-network linderol $(d=3, \mathrm{NC}=54.543 \%)$, momorcharaside $\mathrm{F} 2(d=5, \mathrm{NC}=55.33 \%)$, cis- $\mathrm{N}$-Feruloyltyramine $(d=7, \mathrm{NC}=24.0 \%)$, and beta-sitosterol $(d=10, \mathrm{NC}=31.0 \%)$ had the highest connectivity to major presumed targets and topological measurements (where $d$ is the node degree and $\mathrm{NC}$ is the proportion neighbor connectivity). 
Table 1: Herbal ingredient with significant antidiabetic synergistic effects

\begin{tabular}{lll}
\hline Herbs & Chemical ingredients & Synergy score \\
\hline Zingiber officinale & Beta-sitosterol & $0.4332^{*}$ \\
Zingiber officinale & (S)-10-gingerol & $0.4129^{*}$ \\
Zingiber officinale & [6]-shogaol & 0.4810 \\
Tinospora cordifolia & Berberine bisulfate & 0.3973 \\
Tinospora cordifolia & Cis-N-Feruloyltyramine & $0.3711^{*}$ \\
Tinospora cordifolia & Columbamine & 9.93 \\
Tinospora cordifolia & Oxyberberine & $2.1 \mathrm{E}-02$ \\
Blumea balsamifera & Linderol & $1.0 \mathrm{E}-03$ \\
Blumea balsamifera & Jatrorrhizine & 2.3987 \\
Blumea balsamifera & Magnoflorine & 0.4001 \\
Blumea balsamifera & Palmatine & $0.3071^{*}$ \\
Blumea balsamifera & Blumeatin & 0.3912 \\
Momordica charantia & Momordicoside F2 & 0.4992 \\
Momordica charantia & Goyaglycoside-B & 0.5617 \\
Momordica charantia & Charantoside VII & 0.4110 \\
Momordica charantia & Momordicoside I & $0.3976^{*}$ \\
\hline
\end{tabular}

*berberine-ingredient pairs with significant synergy scores

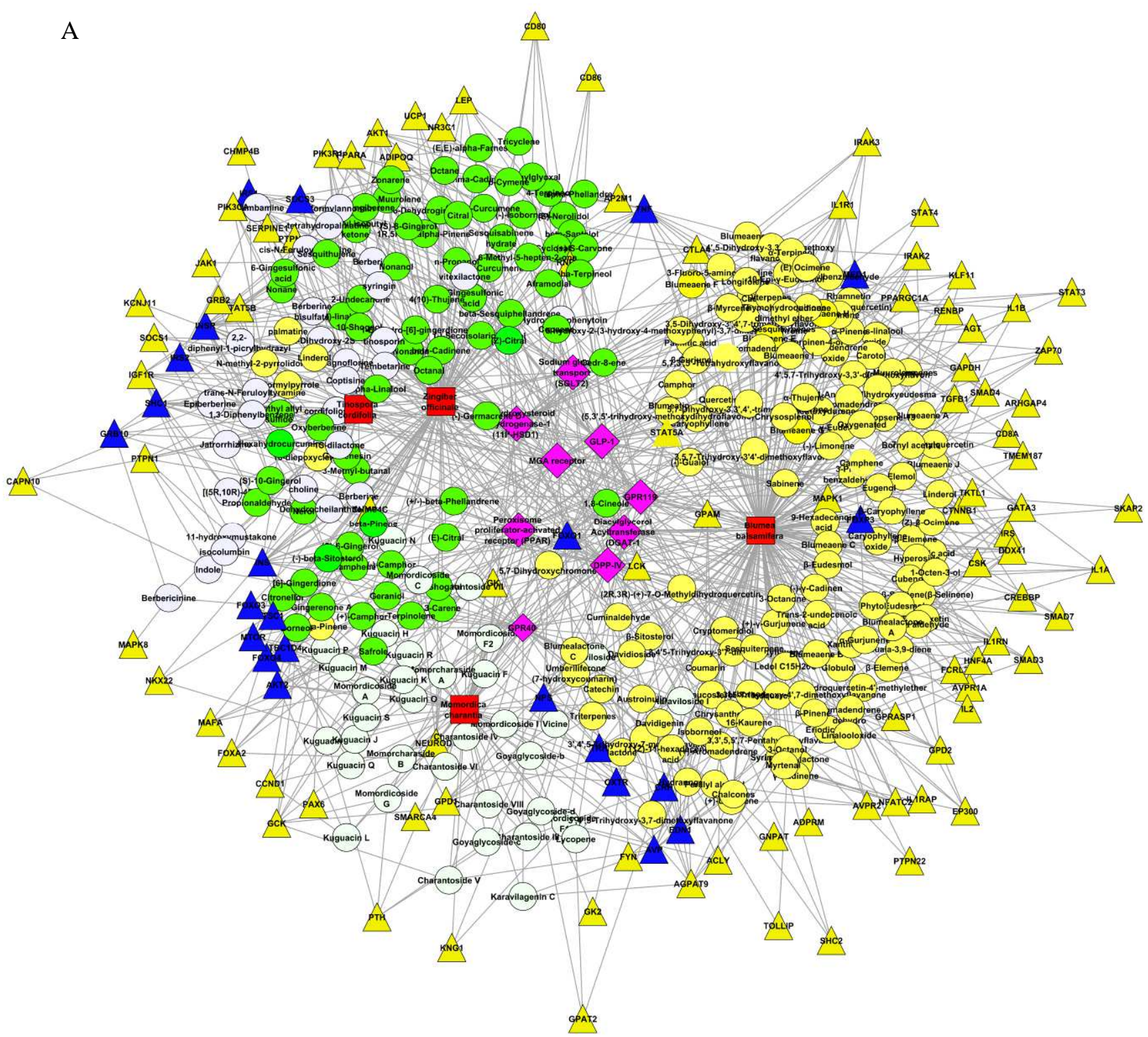




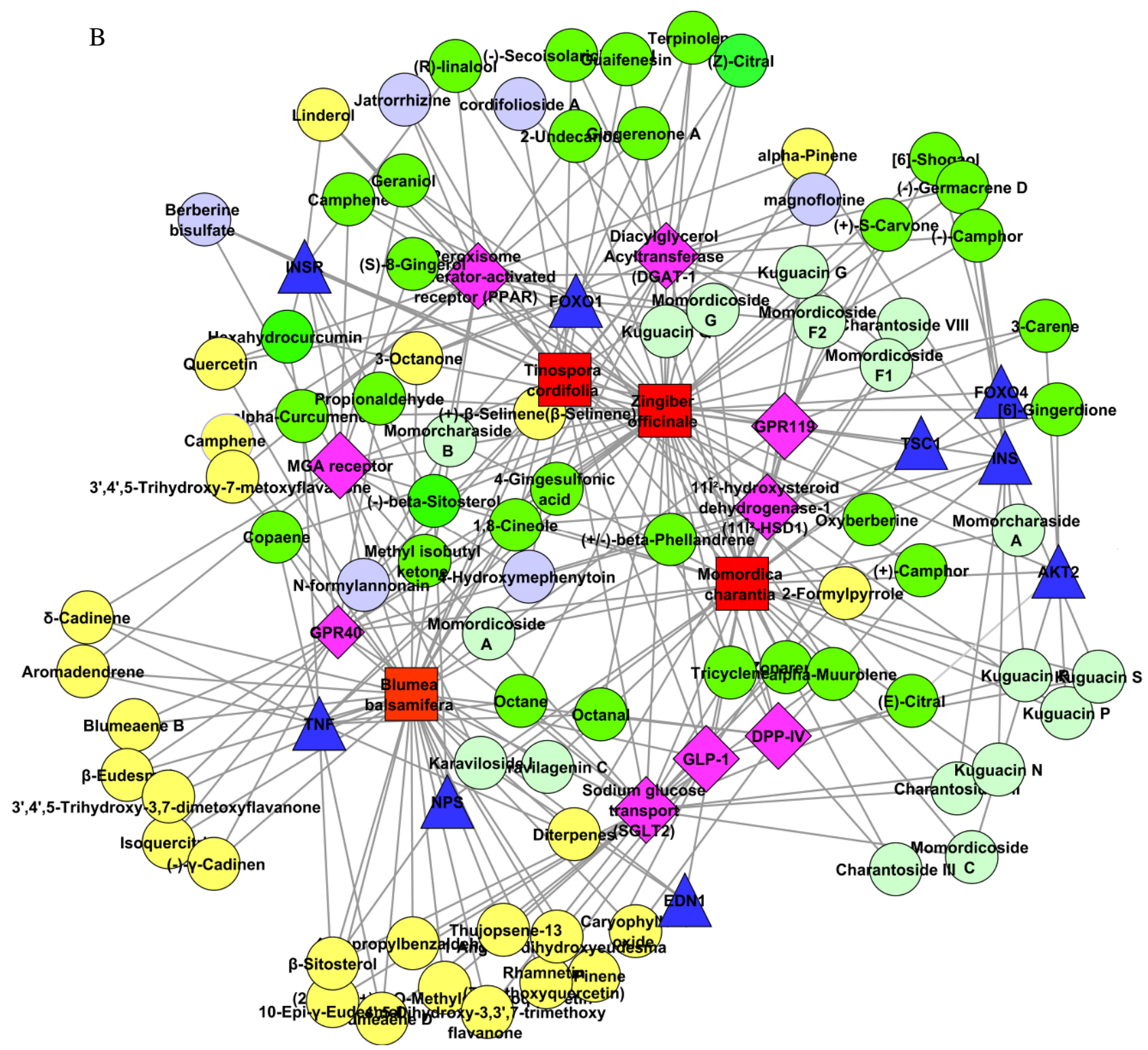

Fig. 3: Network analysis for the identification of the underlying pharmacological mechanisms of the actions of the herbal decoctions acting on T2D. (A) Herb-ingredient-presumed targets-known Type 2 diabetic target network. (B) Major herbs-ingredients-presumed target-known T2D target network visualized by Cytoscape. Edge: interaction between herbal ingredients and their presumed targets; red rectangle node: four herbs including; Tinospora cordifolia, Zingiber officinale, Momordica charantia and Blumea balsamifera; circle nodes: herbal ingredients; brown triangle nodes: presumed targets of herbal ingredients: blue triangle; major presumed shared targets by the four herbs: pink diamond; known therapeutic targets for treatment of type 2 diabetes

\section{Enrichment analysis}

Enrichment analysis (table 2) revealed that herbs major presumed targets were frequently involved in various significant biological processes and pathways such as regulation of insulin receptor signaling pathway (9.16E-07), regulation of glucose metabolic process (2.96E-17), glucose homeostasis (3.75E-09), and regulation of insulin secretion (8.77E-05) and JAK/STAT signaling pathway (3.37E-11). Based on network analysis (fig. 3) major presumed targets including FOXO4, INS, AKT2 and TSC1 genes are linked to momordicoside F2 compound. FOXO4 gene is perceived to regulate insulin signaling pathway. On the other hand, INS gene reduces blood glucose concentration by enhancing cell permeability to monosaccharide's, amino acids, and fatty acids which accelerate glycolysis, the pentose phosphate cycle, and glycogen synthesis in liver [56]. AKT2 genes regulate glucose uptake by mediating insulininduced translocation whereas TSC1 genes act as a tumor suppressor by negatively regulating mTORC1 signaling pathways [57]. Furthermore, FOXO1 and INSR targeted genes are linked to linderol and cis-N-Feruloyltyramine compound, FOXO1 is perceived as the target of insulin signaling and regulates metabolic homeostasis in response to oxidative stress and regulation of glucose metabolism while INSR as a receptor tyrosine kinase mediates the pleiotropic actions of insulin [58]. In addition, NPS INF and EDN1 genes are linked to beta-sitosterol compounds, studies have reported NPS genes to plays an important anorexigenic role while IFN effector genes to triggers interferon-stimulated genes (ISGs) which inhibit virus replication, on the other hand, EDN1 genes have been reported to involve in endothelium-derived vasoconstriction [59]. On this basis, the major presumed targets of Indonesian herbal decoction are significantly associated with these biological processes and pathways might play a role in the understanding mechanism of action of Indonesia herbal decoction in treatment of Type 2 diabetes. 
Table 2: Top 10 significant biological processes and top 10 pathways associated with herbs major presumed targets

\begin{tabular}{|c|c|c|c|}
\hline Terms & Counts & Fold enrichment & P-value \\
\hline \multicolumn{4}{|l|}{ GO Biological process } \\
\hline Regulation of inflammatory response (GO: 0050727) & 12 & 12.19 & $3.96 \mathrm{E}-05$ \\
\hline Regulation of carbohydrate biosynthetic process (GO: 0043255) & 14 & 68.09 & $2.56 \mathrm{E}-18$ \\
\hline Regulation of insulin receptor signaling pathway (GO: 0046626) & 14 & 58.36 & $9.16 \mathrm{E}-07$ \\
\hline Regulation of glucose metabolic process (GO: 0010906) & 17 & 57.07 & $2.96 \mathrm{E}-17$ \\
\hline Regulation of cellular response to insulin stimulus (GO: 1900076) & 14 & 54.35 & $2.22 \mathrm{E}-08$ \\
\hline Regulation of glucose transport (GO: 0010827) & 12 & 48.45 & $1.79 \mathrm{E}-13$ \\
\hline Glucose homeostasis (G0: 0042593) & 11 & 25.87 & 3.75E-09 \\
\hline Regulation of blood pressure (G0: 0008217) & 10 & 24.18 & 9.27E-08 \\
\hline Regulation of insulin secretion (GO: 0050796) & 9 & 18.81 & 8.77E-05 \\
\hline Regulation of lipid metabolic process (GO: 0019216) & 13 & 18.73 & $1.25 \mathrm{E}-09$ \\
\hline \multicolumn{4}{|l|}{ PANTHER Pathways } \\
\hline JAK/STAT signaling pathway (P00038) & 7 & $>100$ & 3.37E-11 \\
\hline Insulin/IGF pathway-mitogen activated protein kinase kinase/MAP kinase cascade (P00032) & 10 & 46.73 & $1.46 \mathrm{E}-07$ \\
\hline Insulin/IGF pathway-protein kinase B signaling cascade (P00033) & 12 & 45.13 & 8.70E-09 \\
\hline PI3 kinase pathway (P00048) & 10 & 28.04 & $1.78 \mathrm{E}-05$ \\
\hline Interleukin signaling pathway (P00036) & 11 & 22.88 & $1.40 \mathrm{E}-08$ \\
\hline PDGF signaling pathway (P00047) & 9 & 18.63 & $2.46 \mathrm{E}-07$ \\
\hline CCKR signaling map (P06959) & 9 & 16.04 & 8.88E-07 \\
\hline Gonadotropin-releasing hormone receptor pathway (P06664) & 11 & 14.44 & 4.71E-08 \\
\hline Wnt signaling pathway (P00057) & 6 & 13.31 & 4.64E-02 \\
\hline Angiogenesis (P00005) & 7 & 12.27 & 2.87E-04 \\
\hline
\end{tabular}

\section{Molecular docking validation}

Molecular docking technique, as a computational structure-based method is a powerful tool in drug discovery and design. This technique can help researchers discover the relationship between the constituents of Indonesian herbal decoctions and network targets [60]. Autodock Vina is high-throughput molecular docking tool with a fast and simple method to rapidly predict the binding affinity of a ligand, based on the geometry of a candidate ligand docked into a target receptor structure using empirical functions [61].

\section{Binding sites profiles}

The four major ligands were selected based on their significant synergy score (table 1) and their topological importance in k-core subnetwork (fig. 3B). Based on Lipinski rules, which state that the drug compound should have a molecular size less than 500 Dalton [62] our docking analysis revealed beta-sitosterol (414 Dalton), cis-NFeruloyl-tyramine (313 Dalton), and linderol (362 Dalton) compounds had a molecular size less than Lipinski limit with only momordicoside F2 (618 Dalton) compound exceeding limit. Furthermore, the binding site profile analysis (fig. 4) revealed that momordicoside F2 had three residues involved in hydrogen bonding (H-bonding) in miglitol (Asp702, Leu727, and Glu719) residues, which switched to hydrophobic interactions in momordicoside F2. Residues of Glu704 also switched its interaction from hydrophobic in miglitol to hydrogen bond in momordicoside F2. For beta-sitosterol, all its interactions with the Maltase-glucoamylase receptor (MGA) were classified as hydrophobic interaction and no any hydrogen bond were involved. The residues involved in hydrophobic interaction in beta-sitosterol included Asp702, Ile725, Lys724, Leu720, and Glu719. For cis-NFeruloyl-tyramine, six residues were involved in the interactions four residues (Glu719, Leu720, Asp702, and Lys724) in hydrophobic interaction whereas two residues in hydrogen bond interaction (Ile725 and Glu704) on the other hand for linderol, only one residue (Glu704) was involved in the hydrophobic interaction. Even though we docked linderol in same targeted grid box used for other three ligands, docking analysis reveals its tendency to bind to the different regions probably due to its small molecular size (362 Dalton) or grid size of targeted receptor thus only Glu704 residue was actively involved in the hydrophobic interaction.

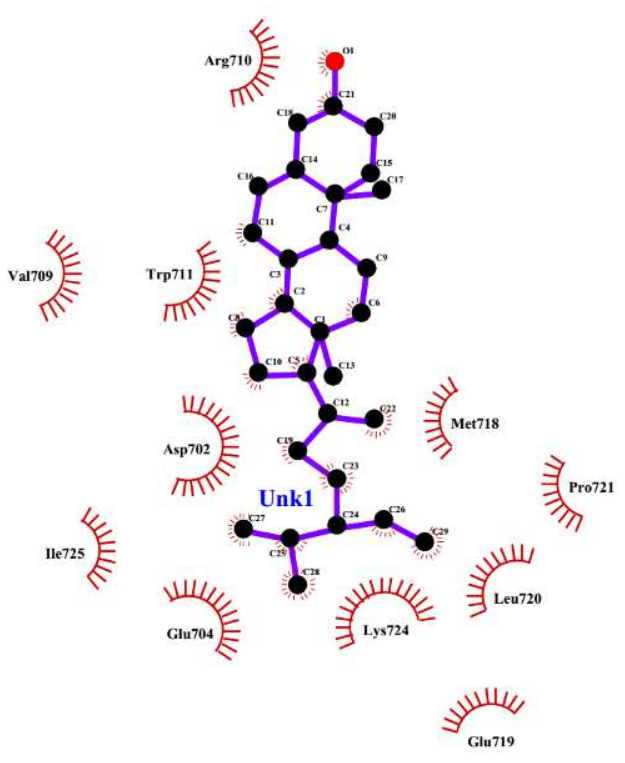

2qmj-betasitosterol

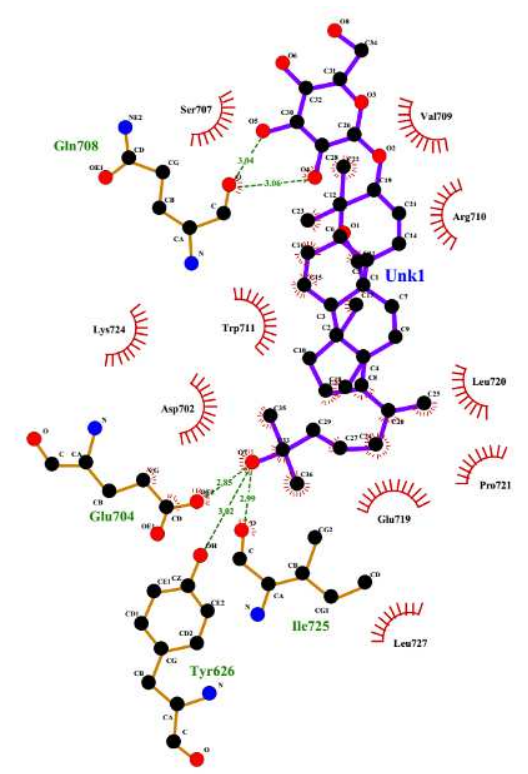

2qmj-momordico 

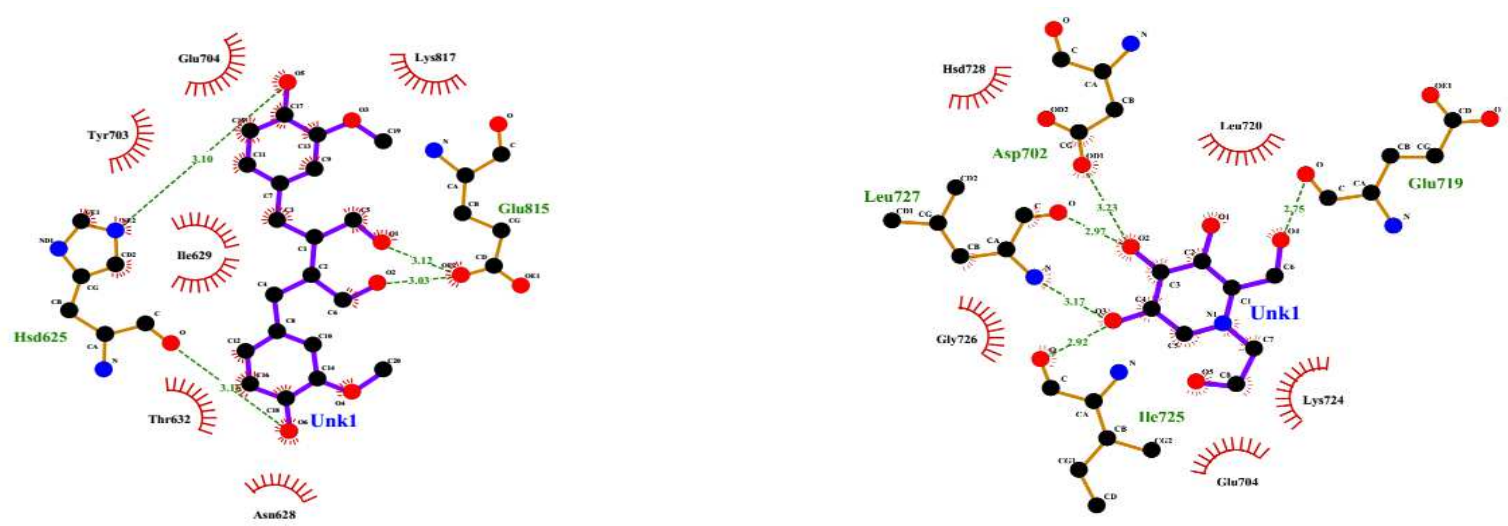

2qmj-linderol

2qmj-miglitol

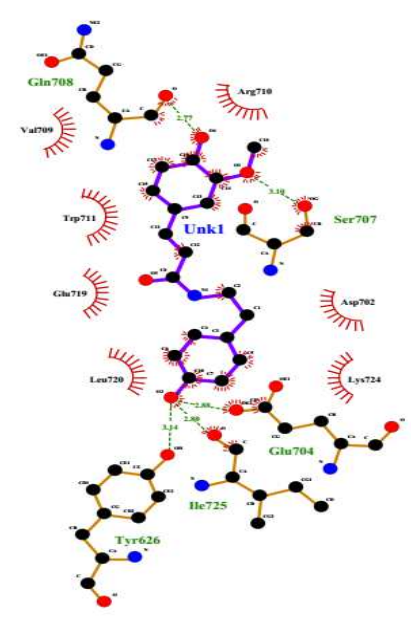

2qmj-cisNFeru

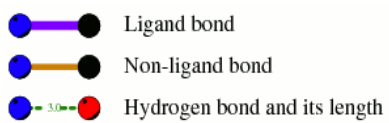

His 53 Non-ligand residues involved in hydrophobic

$\lambda_{\pi, \pi}$ contact(s)

Corresponding atoms involved in hydrophobic contact(s)

Fig. 4: Binding sites similarities of herbs major ingredient (beta-sitosterol, momordicoside F2, cis-N-Feruloyltyramine, and linderol) compared with miglitol visualized by LigPlot+v.1.4 program

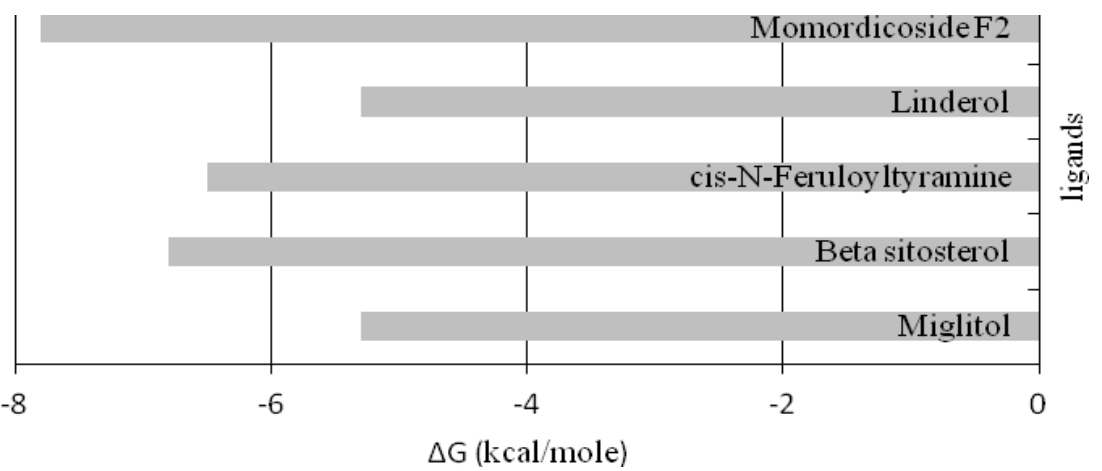

Fig. 5: The binding affinity of herbs major ingredient compounds compared with miglitol drug (control ligand)

Furthermore, when we compare the binding site's similarity of the four ligands to miglitol a patent antidiabetic drug (table 3), momordicoside F2 presents the best binding site similarity(BSS) of $78 \%$ ( 7 out of 9 residues) among the four ligands followed by betasitosterol and cis-N-Feruloyltyramine both scoring $67 \%$ (6 out of 9 residues). Lastly, linderol was the only ligand that scored lowest binding sites similarity $11 \%$ ( 1 out of 9 residues) when compared to miglitol binding sites.

\section{Binding affinity}

The binding affinity of each ligand to Maltase-glucoamylase (MGA) target was assessed to evaluate their Gibbs free energy (fig. 5).

Docking analysis revealed that momordicoside F2 had the best binding affinity $(-7.80 \mathrm{kcal} / \mathrm{mole})$ followed by beta-sitosterol $(-6.80$ $\mathrm{kcal} / \mathrm{mole})$ and cis-N-Feruloyltyramine $(-6.50 \mathrm{kcal} / \mathrm{mole})$. However, linderol turned out to have the same binding affinity with miglitol (- 
Table 3: Binding sites profile analysis of the herbs major ingredients compared with miglitol drug

\begin{tabular}{|c|c|c|c|c|c|c|}
\hline Herb & Ligands & HIR $^{\text {a }}$ & HBR $^{b}$ & $\begin{array}{l}\text { LHFG } \\
\mathrm{c}\end{array}$ & $\begin{array}{l}\text { HBL } \\
\text { d(Å) }\end{array}$ & $\begin{array}{l}\text { BSS e } \\
(\%)\end{array}$ \\
\hline & Miglitol (control drug) & Hsd728,Gly726, Leu720,Glu704, Lys724 & Asp702 & 02 & 3.23 & \\
\hline & & & Leu727 & 02 & 2.97 & 100 \\
\hline & & & Ile725 & 03 & 3.17 & \\
\hline & & & Glu719 & 03 & 2.92 & \\
\hline & & & & 04 & 2.75 & \\
\hline \multirow{2}{*}{$\begin{array}{l}\text { Zingiber officinale } \\
\text { (jahe) }\end{array}$} & Beta-sitosterol & Arg710,Val709, Trp711,Asp702, & & & & \\
\hline & & $\begin{array}{l}\text { Ile725,Glu704, Lys724,Met718, Pro721, Leu720, } \\
\text { Lys724,Glu719 }\end{array}$ & - & - & - & 67 \\
\hline \multirow{5}{*}{$\begin{array}{l}\text { Tinospora cordifolia } \\
\text { (bratawali) }\end{array}$} & cis-N-Feruloyltyramine & Val709,Trp711, Glu719, Leu720, & Gln708 & 04 & 2.77 & \\
\hline & & Arg710,Asp702, & Ser707 & 01 & 3.10 & 67 \\
\hline & & Lys724 & Tyr626 & 02 & 3.14 & \\
\hline & & & Ile725 & 02 & 2.80 & \\
\hline & & & Glu704 & 02 & 2.88 & \\
\hline \multirow{5}{*}{$\begin{array}{l}\text { Momordica charantia } \\
\text { (pare) }\end{array}$} & Momordicoside F2 & Ser707,Lys724, & Gln708 & 05 & 3.04 & \\
\hline & & Trp711, Asp702, Val709,Arg710, & Glu704 & 04 & 3.06 & 78 \\
\hline & & Leu720, Pro721, Glu719, Leu727 & Tyr626 & 07 & 2.85 & \\
\hline & & & Ile725 & 07 & 3.02 & \\
\hline & & & & 07 & 2.99 & \\
\hline \multirow{4}{*}{$\begin{array}{l}\text { Blumea balsamifera } \\
\text { (sembung) }\end{array}$} & Linderol & Glu704,Tyr703, Lys817, Ile629, Thr632, Asn628 & & 03 & 3.10 & \\
\hline & & & Hsd 625 & 06 & 3.16 & 11 \\
\hline & & & Glu815 & 01 & 3.12 & \\
\hline & & & & 02 & 3.03 & \\
\hline
\end{tabular}

a: HIR hydrophobic interaction residue; b: HBR hydrogen bonding residues; c: LHFG ligand hydrogen bonding group; d: HBL hydrogen bond length; e: BSS binding site similarity

\section{CONCLUSION}

This paper presents a comprehensive systematic approach integrating target profile analysis, synergy prediction, network analysis, and target validation to reveal the relationships between ingredients contained in the four Indonesia herbal decoctions and their presumed targets and Type 2 diabetes related pathway systems. From our results target profile analysis via Markov clustering (MCL) provided clues to possible antidiabetic ingredients in the four herbs to investigate their pharmacological mechanisms to the treatment of Type 2 diabetes with an overall success rate of 98.58\%. Synergy prediction by network target based identification of multicomponent synergy revealed there were herbal ingredients with significant antidiabetic synergetic effects.

The multi-level and integrated network targets of herbs, their presumed targets and known Type 2 diabetes therapeutic targets revealed the herbs major ingredients and their presumed targets acting on Type 2diabetes therapeutic targets. Enrichment analysis revealed major presumed targets were frequently involved in significant biological processes and pathways related to the progression of Type 2 diabetes (T2D). For molecular docking validation momordicoside F2 (78\%), beta-sitosterol (67\%) and cis$\mathrm{N}$-Feruloyltyramine (67\%) presented higher binding site similarity when compared with miglitol drug indicating their potential to develop antidiabetic drugs although linderol had low binding site similarity probably due to its smaller molecular size (362 Dalton) or the size of grid box used for docking all the ligands. Furthermore, all the four ligands indicated higher binding affinity and inhibitory properties to Maltase-glucoamylase (MGA) receptor. Thus the docking results reveal the pharmacological mechanism of action of Indonesia herbal decoction in treatment of Type 2diabetes with momordicoside F2, beta-sitosterol, cis-N-Feruloyltyramine and linderol indicating the potential of curing Type 2 diabetes disease (T2D). Since our preliminary study was purely based on network pharmacology techniques and bioinformatics analysis, further experimental studies are required to test the hypotheses.

\section{CONFLICTS OF INTERESTS}

The authors declare that there is no conflict of interests regarding the publication of this paper.

\section{REFERENCES}

1. Marre M, Penfornis A. GLP-1 receptor agonists today. Diabetes Res Clin Pract 2011;93:317-27.

2. Parkes DG, Mace KF, Trautmann ME. Discovery and development of exenatide: the first antidiabetic agent to lever age the multiple benefits of the in cretin hormone, GLP-1. Expert Opin Drug Discov 2014;8:219-44.

3. Shaw JE, Sicree RA, Zimmet PZ. Global estimates of the prevalence of diabetes for 2010 and 2030. Diabetes Res Clin Pract 2010;87:4-14.

4. Jeffrey S, Flier. Hormone resistance in diabetes and obesity: insulin, leptin, and FGF21. Yale J Biol Med 2012;85:405-14.

5. Leahy L, Hirsch BI, Peterson KA, Schneider D. Targeting betacell function early in the course of therapy for type 2 diabetes mellitus. J Clin Endocrinol Metab 2010;95:4206-16.

6. Ku CR, Lee HJ, Kim SK. Resveratrol prevents streptozotocin induced diabetes by inhibiting the apoptosis of pancreatic betacell and the cleavage of poly (ADP-ribose) polymerase. Endocr J 2012;59:103-9.

7. Kannan, Arshad, Kumar S. A study on drug utilization of oral hypoglycemic agents in type-2 diabetic patients. Asian J Pharm Clin Res 2011;4:60-4.

8. Jovanovic L, Hassman DR, Gooch B. Treatment of type 2 diabetes with a combination regimen of repaglinide pluspioglitazone. Diabetes Res Clin Pract 2004;63:127-34.

9. Howlett HCS, Bailey CJ. A risk-benefit assessment of metformin in type 2 diabetes mellitus. Drug Safety 1999;20:489-503.

10. Gastaldelli A, Ferrannini E, Miyazaki Y, Matsuda M, Mari A, DeFronzo RA. tiazolidinediones improve beta cell function in type 2 diabetic patients. Am J Physiol 2007;292:E871-83.

11. Vengurlekar S, Shukla P, Patidar P, Bafna R, Jain S. Prescribing pattern of antidiabetic drugs in indore city hospital. Indian J Pharm Sci 2008;70:637-40. 
12. Karunrat T, Walaya J. The pharmacokinetics of 2 doses levothyroxine treatment in athyreotic patients. Int J Appl Pharm 2016;4:66-8.

13. Kumar KS, Sreeramya G, Krishna KM, Nalini K, Kiranmai N, Vasavi P. Drug use pattern study of antidiabetics in type 2 diabetes mellitus at a tertiary care hospital in Tenali, Andhra Pradesh. Int J Invent Pharm Sci 2013;1:162-6.

14. Ciero LT, Yenshou, Lin AP, Yi C, Shao CC, Wen CY. Herbal therapies for type 2 diabetes mellitus: chemistry, biology, and potential application of selected plants and compounds. J Evidence-Based Complementary Altern Med 2013:1-33. Doi:10.1155/2013/378657

15. Zhang XJ, Deng YX, Shi QZ, He MY, Chen B, Qiu XM. Hypolipidemic effect of the Chinese polyherbal Huanglian Jiedu decoction in type 2 diabetic rats and its possible mechanism. J Phymed 2014;21:615-23.

16. Yuxin P, Dan W, Zuowang F, Xiaolu C, Fulai Y, Xuan H, et al. Blumea balsamifera phytochemical and pharmacological. Mol Rev 2014;19:9453-77.

17. Dong H, Wang N, Zhao L, Lu F. Berberine in the treatment of type 2 diabetes mellitus: a systemic review and meta-analysis. J Evidence-Based Complementary Altern Med 2012. Doi:10.1155/2012/591654.

18. Reddy NM, Rajasekhar R. Tinospora cordifolia chemical constituents and medicinal properties: a review. Scholars Acad J Pharm 2015;4:364-9.

19. Chakraborty D, Mukherjee A, Sikdar S. [6]-gingerol isolated from ginger attenuates sodium arsenite induced oxidative stress and plays a corrective role in improving insulin signaling in mice. Toxicol Lett 2010;210:34-43.

20. Prabhakar PK, Doble M. Mechanism of action of natural products used in the treatment of diabetes mellitus. Chin J Integr Med 2011;17:563-74.

21. Biswal S, Sahoo U, Sethy S, Kumar HKS, Banerjee M. Indole: the molecule of diverse biological activities. Asian J Pharm Clin Res 2012;5:1-6.

22. Thomas N, Zachariah SM. Pharmacological activities of chromene derivatives: an overview. Asian J Pharm Clin Res 2011;6:11-5

23. Zhang B, Wang X, Li S. An integrative platform of TCM network pharmacology and its application on an herbal formula. J Evidence-Based Complementary Altern Med 2013. http://dx.doi.org/10.1155/2013/456747

24. Li S, Zhang B, Jiang D, Wei Y, Zhang N. Herb network construction and co-module analysis for uncovering the combination rule of traditional Chinese herbal formulae. BMC Bioinfo 2010;11 Suppl 11:S6.

25. Gustafsson M, Nestor CE, Zhang H, Barabási AL, Baranzini S. Modules, networks and systems medicine for understanding disease and aiding diagnosis. Genome Med 2014;6:82-6.

26. Xiujuan W, Natali G, Haiyuan Y. Network-based methods for human disease gene prediction. Briefings Funct Genomics 2011;10:280-93.

27. Wei L, Aiping W, Matteo P, Xiaofan W. Integrative analysis of human protein, function and disease networks. Nat Sci Rept 2015. Doi:10.1038/srep14344

28. Li S, Zhang B, Zhang N. Network target for screening synergistic drug combinations with application to traditional Chinese medicine. BMC Syst Biol 2011;5(Suppl 1):S10.

29. Liang X, Li H, Li SA. Novel network pharmacology approach to analyses traditional herbal formulae: the Liu-Wei-Di-Huang pill as a case study. Mol Biosyst 2014;10:1014-22.

30. Wang Y, Xiao J, Suzek TO, Zhang J, Wang J, Bryant SH. PubChem: a public information system for analyzing bioactivities of small molecules. Nucleic Acids Res 2009;37:W623-33.

31. Barrett T, Wilhite SE, Ledoux P, Evangelista C, Kim IF, Tomashevsky M. NCBI GEO: archive for functional genomics data sets-update. Nucleic Acids Res 2013;41:D991-5.

32. Hamosh A, Scott AF, Amberger JS, Bocchini CA, McKusick VA. Online mendelian inheritance in man (OMIM), a knowledge base of human genes and genetic disorders. Nucleic Acids Res 2005;33:D514-17.

33. Wishart DS, Knox C, Guo AC, Cheng D, Shrivastava S, Tzur D, et al. DrugBank: a knowledge base for drugs, drug actions and drug targets. Nucleic Acids Res 2008;36:D901-6.
34. Chen JY, Mamidipalli S, Huan T. HAPPI: an online database of comprehensive human annotated and predicted protein interactions. BMC Genomics 2009;34:D140-44.

35. Keshava PS, Goel R, Kandasamy K, Keerthikumar S, Kumar S, Mathivanan S. Human protein reference database. Nucleic Acids Res 2009;37:D767-72.

36. Brown KR, Jurisica I. Online predicted human interaction database. Bioinformatics 2005;21:2076-82.

37. Ceol A, Chatr-Aryamontri A, Armstrong J, Woollard P. MINT, The molecular interaction database. Nucleic Acids Res 2010;38:D532-39.

38. Nacher JC, Schwartz JM. A global view of drug-therapy interactions. BMC Pharmacol 2008;8:5-14.

39. Korcsmáros T, Szalay MS, Böde C, Kovács IA, Csermely P. How to design multi-target drugs: target search options in cellular networks. Expert Opin Drug Discovery 2007;2:1-10.

40. Arrell DK, Terzic A. Network systems biology for drug discovery. Clin Pharmacol Ther 2010;88:120-5.

41. Hwang WC, Zhang A, Ramanathan M. Identification of information flow modulating drug targets: a novel bridging paradigm for drug discovery. Clin Pharmaco Ther 2008;84:563-72.

42. Shannon P, Markiel A, Ozier O, Baliga NS, Wang JT, Ramage D, et al. Cytoscape: a software environment for integrated models of biomolecular interaction networks. Genome Res 2013;3:2498504.

43. Li S, Zhang B. Traditional Chinese medicine network pharmacology: theory, methodology and application. Chinese J Natur Med 2013;11:110-20.

44. Wuchty S, Almaas E. Evolutionary cores of domain cooccurrence networks. BMC Evol Biol 2005;5:24.

45. Mi H, Tomas P. PANTHER pathway: an ontology-based pathway database coupled with data analysis tools. Methods Mol Biol 2009;563:123-40.

46. Huang DW, Sherman BT, Lempicki RA. Systematic and integrative analysis of large gene lists using DAVID bioinformatics resources. Nature Protocols 2009;4:44-57.

47. Trott 0 , Olson AJ. AutoDock Vina: improving the speed and accuracy of docking with a new scoring function, efficient optimization and multithreadine. J Comput Chem 2010;31:455-61.

48. Morris GM, Huey R, Lindstrom W, Sanner MF, Belew RK, et al. AutoDock4 and AutoDockTools4:automated docking with selective receptor flexibility. J Comput Chem 2009;16:85-91.

49. Laskowski RA, Swindells MB. LigPlot+: multiple ligand-protein interaction diagrams for drug discovery. J Chem Inf Model 2011;51:2778-86.

50. Zhang B, Wang X, Li S. An integrative platform of TCM network pharmacology and its application on herbal formula, Qing-LuoYin. J Evidence-Based Complementary Altern Med 2013. http://dx.doi.org/10.1155/2013/456747

51. Xie W, Gu D, Li J, Cui K, Zhang Y. Effects and action mechanisms berberine and Rhizoma coptidis on gut microbes and obesity in high-fat diet-fed C57BL/6J mice. PloS one 2011. http://dx.doi.org/10.1371/journal.pone.0024520

52. Hadimani MB, Purohit MK, Vanampally C. Guaifenesin derivatives promote neurite outgrowth and protect diabetic mice from neuropathy. J Med Chem 2013;56:5071-8.

53. Yin J, Gao Z, Liu D, Liu Z, Ye J. Berberine improves glucose metabolism through induction of glycolysis. Am J Physiol Endocrinol Metab 2008;294:E148-56.

54. Bliss CI. The calculation of microbial assays. Bacteriol Rev 1956;20:243-58.

55. Diana S, Kara N, Eleanor P, Ji EC, Hertzel CG. The effect of oral antidiabetic agents on A1C levels. A systematic review and meta-analysis. BMJ Open Diabetes Res Care 2010;33:1859-64.

56. Da Silva Xavier G, Varadi A, Ainscow EK, Rutter GA. Regulation of gene expression by glucose in pancreatic $\beta$-cells (MIN6) via insulin secretion and activation of phosphatidylinositol $3^{\prime}$ kinase. J Biol Chem 2000;275:36269-77.

57. O'Neill LA, Bowie AG. Sensing and signaling in antiviral innate immunity. Curr Biol 2010;20:R328-33. 
58. Moore PC, Ugas MA, Hagman DK, Parazzoli SD, Poitout V. Evidence against the involvement of oxidative stress in fatty acid inhibition of insulin secretion. Diabetes 2004;53:2610-6.

59. Borden EC, Williams BR. Interferon-stimulated genes and their protein products what and how? J Interferon Cytokine Res 2011;31:1-4.

60. Zhang GB, Li QY, Chen QL, Su SB. Network pharmacology: a new approach for Chinese herbal medicine research. J Evidence Based Complementary Altern Med 2013. http://dx.doi.org/ 10.1155/2013/621423.

61. Wang RZ, Lin DQ, Tong HF, Yao SJ. Molecular insights into the binding selectivity of a synthetic ligand DAAG to Fc fragment of IgG. J Mol Recognit 2014;27:250-9.
62. Lipinski CA, Lombardo F, Dominy BW, Feeney PJ. Experimental and computational approaches to estimate solubility and permeability in drug discovery and development settings. Adv Drug Delivery Rev 2001;46:3-29.

\section{How to cite this article}

- $\quad$ Peter Juma Ochieng, Wisnu Ananta Kusuma, Mohamad Rafi, Tony Sumaryada. Deciphering the action mechanism of Indonesia herbal decoction in the treatment of type II diabetes using a network pharmacology approach. Int J Pharm Pharm Sci 2017;9(3):243-253. 